\title{
LiDAR Based Multi-Robot Cooperation for the 3D Printing of Continuous Carbon Fiber Reinforced Composite Structures
}

\author{
Nanya $\mathrm{Li}^{1}$, Guido Link, Junhui Ma, John Jelonnek \\ Institute for Pulsed Power and Microwave Technology, Karlsruhe Institute of \\ Technology, Eggenstein-Leopoldshafen, 76344, Germany
}

\begin{abstract}
D printing of lightweight continuous carbon fiber reinforced plastics (CCFRP) in three dimensions changes the traditional composite manufacturing processes. The continuous carbon fibers reinforced plastic filament can be printed along the load transmission path and significantly improve the strength of composite structures. Compared to the three-axis computer numerical controlled (CNC) machine based printing process, industrial robots provide the possibility to manufacture complex, spatial and large-scale composite structures. Here, the concept to use multi-robot to print complex spatial CCFRP components simultaneously has been presented. More than one 6 degrees of freedom industrial robots can cooperate with each other and solve the contradiction between structural complexity and printing reachability. During the printing process, the deformation of composite structures may happen, especially for the self-supporting components. Thus, in this paper, a Light Detection and Ranging (LiDAR) method is introduced to detect the deformation of printed composite structure and the movements of two UR robots. To obtain the point clouds of the printed structure, a LiDAR camera D435i has been installed on one robot. A new approach by combining coordinate transformation and iterative-closest-point (ICP) algorithm has been developed to merge the point clouds collected from different shooting angles of the camera.
\end{abstract}

Keywords. Multi-Robot printing, 3D printing, Microwave heating, LiDAR detection, Continuous fiber reinforced composites.

\section{Introduction}

Since the lightweight continuous carbon fiber reinforced thermoplastics (CCFRP) have outstanding strength to weight ratio, long service life, and good damage resistance, the CCFRP structures have been used in various industrial applications. A prominent example is the Airbus 350XWB aircraft that consist of $52 \mathrm{wt} . \%$ continuous carbon fiber reinforced composites in the airframe and enables it to carry more passengers, burn less fuel, and fly farther [1]. Another case is the automobiles, which every $10 \%$ reduction in weight can cut fuel consumption by about $7 \%$. Despite being half the weight of steel, CCFRP is four times stronger and have the potential to reduce automobiles' weight by up to $60 \%$ [3]. 3D printing technologies eliminated the high-cost forming tools and complicated multi-step manufacturing processes [2], the printing with continuous fiber reinforcements shows huge potential to increase the mechanical properties of CCFRP

\footnotetext{
${ }^{1}$ Corresponding author: Nanya Li, Institute for Pulsed Power and Microwave Technology, Karlsruhe Institute of Technology, Eggenstein-Leopoldshafen, 76344, Germany, E-mail: nanya.li@kit.edu
} 
[3]. Meanwhile, the 3D printing of CCFRP can manipulate the fiber orientation and material distribution to allow for maximum weight reduction [4].

Industrial robots have a high degree of freedom, enabling the construction of complex objects without supporting structures [5]. For example, De Backer et al. [6] studied the multi-material fused filament fabrication with continuous fiber reinforcements by using an industrial robot. Generally, the deformation of printed continuous fiber reinforced polymer composite is induced by temperature variation and external forces. To measure the deformation of the printed composite structure, Light Detection and Ranging (LiDAR) camera has been applied, which can scan the structure and surrounding environment without contact. Kabustan et al. [7] created a sensor system for autonomous working industrial robots and built the entire digital space around the robots. Cueva et al. [8] showed how to use Kinect v2 to control an industrial robot by aiding disabilities into daily working activities through gesture recognition. Wang et al. [9] presented human-robot collaboration in the manufacturing context using Kinect camera and aimed to realize a shared workspace where humans can work side by side with robots.

For the $3 \mathrm{D}$ printing of CCFRP, the continuous carbon fibers can be printed along the load transmission path. However, the complex composite structure, e.g. selfsupporting lattice truss and bionic component, reduces the reachability of the printing head. In some areas, the continuous carbon fibers need to be cut and the structure will be reconnected at these joints. By using multi-robot and developing cooperation strategies of the robots, it shows promising advantages to manufacture complex CCFRP components. In this paper, the multi-robot cooperation strategy for $3 \mathrm{D}$ printing of CCFRP has been presented. The LiDAR cameras of Realsense D435i has been employed. The camera will be installed on one robot to scan the printed structure. Thus, the point clouds captured by the D435i camera can indicate the deformation of the printed CCFRP and the printing path of another robot will be revised to ensure the filament can be printed on the right position of the joints.

\section{Multi-Robot cooperation strategy for 3D printing}

The multi-robot cooperation strategy for the 3D printing process is shown in Figure 1. According to the complexity of the CCFRP structure, the robots have different tasks. For example, robot 1 has an in-nozzle impregnation printing head, which can mix the continuous carbon fiber and thermoplastic material in the nozzle during printing. Layerby-layer printing can fabricate ground plate and surface laminates. The printed laminates can cover the CCFRP skeletons, form protection, and aerodynamic contour. As shown in Figure 1, robot 2 prints large-scale free-standing CCFRP filaments. The large-scale CCFRP filaments could be produced before the printing process and be printed by advanced heating methods, e.g. microwave heating. The microwaves have the benefits of instantaneous, selective and volumetric heating. Other robots can be managed to take different tasks depends on the printing objectives. In order to control the printing quality, LiDAR scanning and detecting are vital to monitor the printed CCFRP structures. 


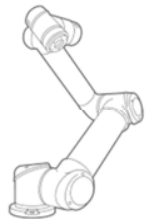

Robot 1

Task 1

Layer-by-layer printing, small diameter CCFRP filaments

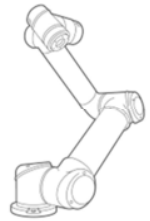

Robot 2

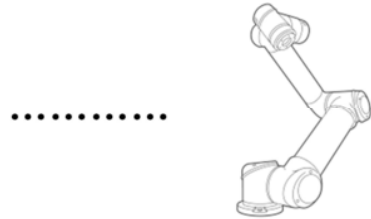

Robot $n$ Task 2 Task n

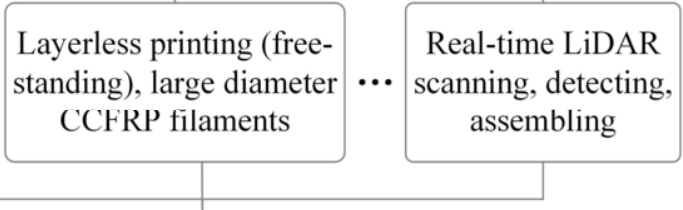

High-performance, complex and lightweight CCFRP structures

Figure 1. Multi-Robot cooperation strategy for 3D printing process

\section{3D printing of CCFRP using the two UR robots platform}

The digital models of the 3D printing platform with two UR robots have been established, as shown in Figure 2. UR10e and UR 16e can lift up to $10 \mathrm{~kg}$ and $16 \mathrm{~kg}$ printing head with posture repeatability of $\pm 0.05 \mathrm{~mm}$. Two robots have been installed on the pillars and the distance between the two robots is about $1.3 \mathrm{~m}$. RoboDK has been used to connect and control the two robots remotely. The programming and simulation tasks can be implemented in RoboDK, offline and online control are both available with the Ethernet connection between the robots and RoboDK. The two printing heads are properly aligned with the end effector of the robots, and its tool point is shifted to the position of the printing nozzle. The detailed information of the conventional and microwave heating assisted printing heads are shown in Figure 2. The Cartesian coordinate system is located on the printed head and the printing bed has been assigned. Collision detection allows users to check collisions between all objects in the digital environment based on the programmed robot paths. In case of collisions between robots, printing heads and the supporting platform have been detected during the simulation process, the collision areas will be highlighted for the user to adjust the printing path.

The printing paths of the two UR robots have been designed in MATLAB and generated as a G-code file. Then the G-code file is transformed to UR robot control language in RoboDK. The designed printing paths of CCFRP parts for two robots are shown in Figure 4. The conventional printing head can only print layer-wise parts as the hotend has limited heating capacity. Thus, the path of the spiral curved hollow skeleton is generated for the conventional printing head, which has $15 \mathrm{~mm}$ diameter and $0.13 \mathrm{~mm}$ height of each layer, as shown in Figure 3 (a). The designed free-standing lattice truss has a length and width of $200 \mathrm{~mm}$, and $20 \mathrm{~mm}$ height, as shown in Figure 3 (b). The microwave heating $(2.45 \mathrm{GHz})$ assisted printing head has been used to print the freestanding lattice truss and about $18 \mathrm{~W}$ microwave power is arranged to heat the preproduced CCFRP filament $(0.45 \mathrm{~mm}$ diameter $)$ to $220^{\circ} \mathrm{C}$. Then the pre-heated filament goes through the extrusion nozzle and infiltrated by polyamide (PA6) materials. Both of the two CCFRP structures are reinforced by $1 \mathrm{~K}$ continuous carbon fibers. 

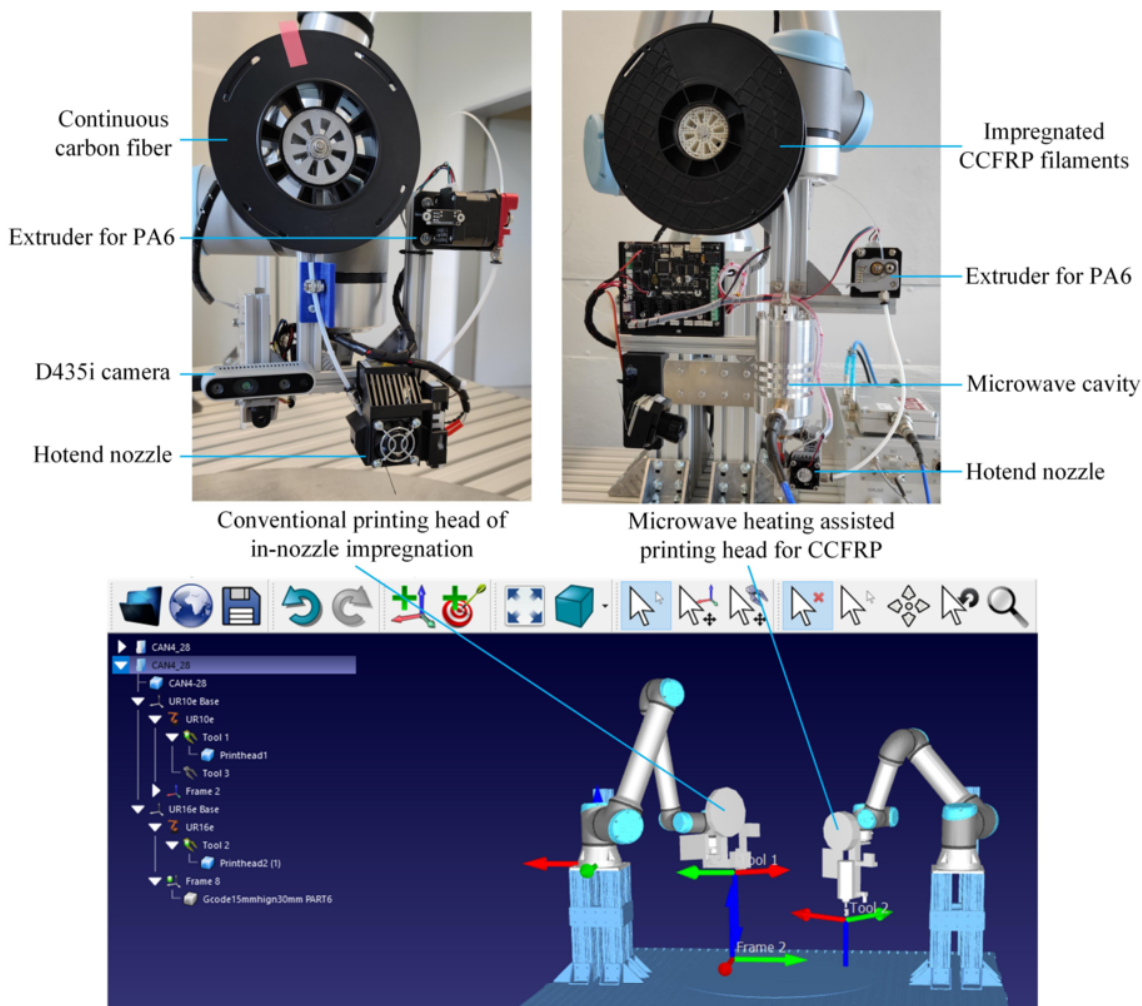

$3 \mathrm{D}$ printing platform of two UR robots

Figure 2. 3D printing platform of CCFRP and the printing heads installed on two UR robots

a)
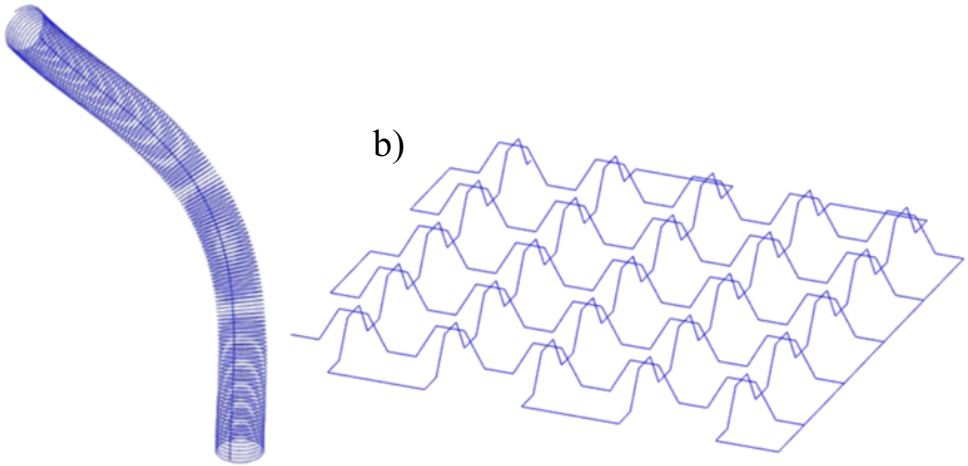

Figure 3. Designed printing paths of CCFRP parts for two robots, a) spiral curved hollow skeleton, b) free-standing lattice truss

The printing speed of the two CCFRP structures is $3 \mathrm{~mm} / \mathrm{s}$ and the nozzle temperature has been set to $240{ }^{\circ} \mathrm{C}$. In addition, compressed airflow is used to rapidly decrease the temperature of the CCFRP material printed by the microwave heating assisted printing head. The printed two structures are shown in Figure 4. Due to the influence of gravity, the spiral curved hollow skeleton has a large deformation. For the 
free-standing lattice truss, the external force is more complex and the shape distortion needs to be controlled. However, the measuring accuracy of the D435i camera is about $2 \mathrm{~mm}$ in a detecting distance of $20 \mathrm{~cm}$. The $1.0 \mathrm{~mm}$ diameter lattice truss cannot be detected by the camera. Thus, in the LiDAR-based scanning section, the deformation of the hollow skeleton has been measured.
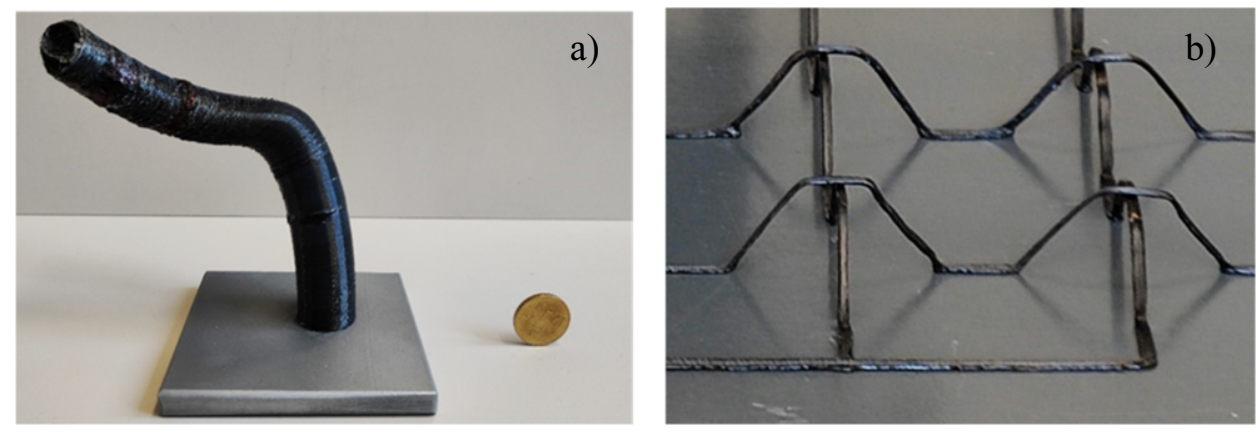

Figure 4. 3D printed a) spiral curved hollow skeleton, and b) free-standing lattice truss

\section{LiDAR based scanning of 3D printed CCFRP structure}

The D435i LiDAR camera used in this study is based on structured light technology. The depth map of the scanning object can be established by measuring the properties of the reflected light from different points on the target. Due to the camera has been installed on the printing head, the relative coordinates of the camera can be obtained from the robot control system. Therefore, the coordinate transformation method is applied to transform all of the point clouds data to the same Cartesian coordinate system firstly. However, the transformed point clouds cannot overlap each other exactly. Because the internal original coordinate point in the D435i camera is not accurate and the measured relative position between the camera and the robot system has errors. Thus, a new approach by combining coordinate transformation and iterative-closest-point (ICP) algorithm has been developed to merge the acquired point clouds.

First, a reference coordinate system is created in RoboDK and the internal coordinate system of the camera has been set as the tool point of the robot. Then the robot has been programmed to move around the printed object and different frames of the point clouds are collected during the movement of the robot. After the point cloud data has been collected, a motion program is generated in RoboDK for the target points that have just been moved. The position of the camera is obtained and the posture variable from the robot program is formatted as $\mathrm{p}[\mathrm{x}, \mathrm{y}, \mathrm{z}, \mathrm{Rx}, \mathrm{Ry}, \mathrm{Rz}]$, where the distances of $\mathrm{x}, \mathrm{y}$ and $\mathrm{z}$ are measured in meters and the rotation angles of $\mathrm{Rx}, \mathrm{Ry}$ and $\mathrm{Rz}$ are measured in radians. For example, the two posture positions 1 and 2 are obtained in equations (1) and (2), then the relative displacement can be calculated by $\triangle x=x 2-$ $x 1, \triangle y=y 2-y 1, \Delta z=z 2-z 1$, the same for the rotation angles.

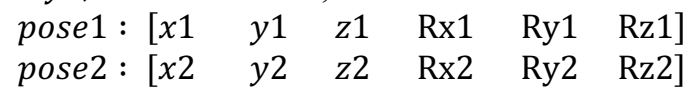

To simplify the LiDAR-based measurement, the robot moves in a quarter circle in front of the printed skeleton, and the distance between the D435i camera and the object is about $25 \mathrm{~cm}$. In the case of the camera rotates along the $\mathrm{Y}$-axis of the camera, which is the axis pointing straight to the printing bed, the rotation angle is defined as $\beta$. 
According to the rotation and displacement of the camera, the coordinate transformation matrix is illustrated in equation (3). Therefore, the point clouds (PTC) will be transformed to the same reference coordinate system by using the matrix, as shown in Figure 5 .

$$
T=\left[\begin{array}{cccc}
\cos \beta & 0 & \sin \beta & 0 \\
0 & 1 & 0 & 0 \\
-\sin \beta & 0 & \cos \beta & 0 \\
\Delta x & \Delta y & \Delta z & 1
\end{array}\right]
$$

As discussed previously, after the coordinate transformation, the iterative-closestpoint algorithm is employed to increase the overlap ratio of the PTC. The flow chart of the reconstruction procedure of the PTC is shown in Figure 5. In principle, the ICP algorithm creates a transformation matrix by iteratively looking for the correspondence relationship of the points in a certain three-dimensional area between two PTCs. By multiplying the generated matrix, the two PTCs which already shifted to the same coordinate system can be precisely overlapped. In detail, the tangents on the target points are calculated to find the closest point in the source PTC, and then a least-squares regression method has been applied to create the transformation matrix $\mathrm{R}$ and transform the target PTC, as illustrated in Figure 5.

Coordinate system transformation

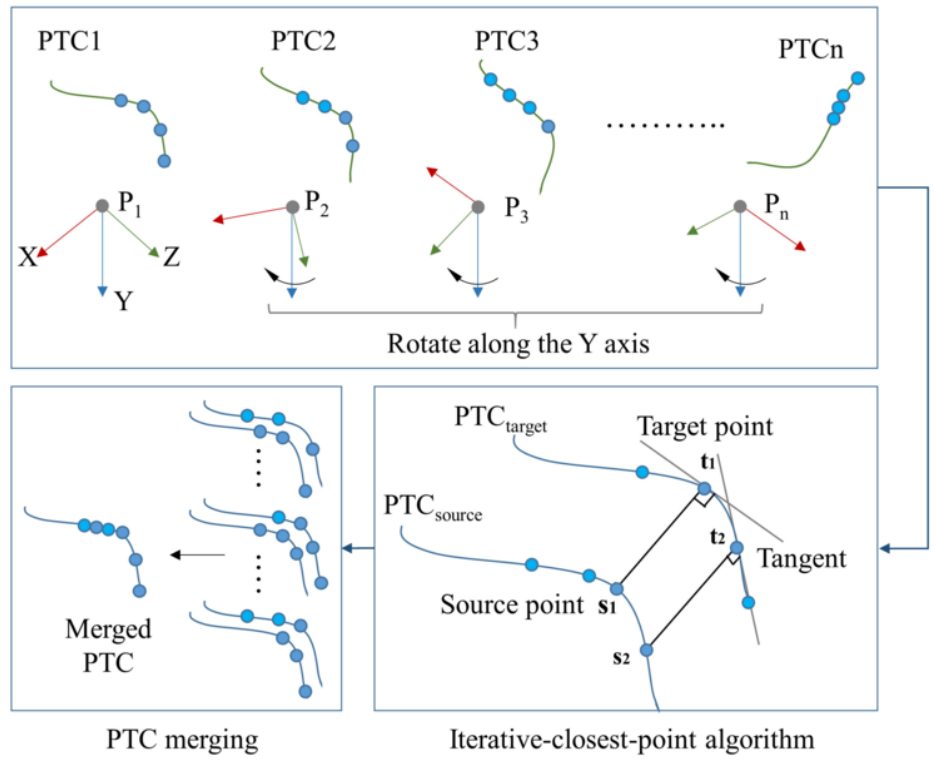

Figure 5. Flow chart of the 3D reconstruction method of the point clouds (PTC)

$\mathrm{R}$ is a transformation matrix of four times and the same as the T matrix. The ICP algorithm, on the other hand, has a limitation to merge the PTCs. In case of the two PTCs cannot find enough feature points that are the same area on the scanning object shared by the two PTCs, the ICP algorithm cannot merge them together. Therefore, the nearby PTCs scanned with small movement and rotation angle of the camera are combined and then all of the PTC pairs are merged together. This merging process has the same procedure as the bubble sort algorithm. For each of the PTC, two transformation matrixes 
$T$ and $R$ are applied to convert the PTC to the right position. The equation can be expressed as follows:

$$
Q^{\prime}=Q * T * R
$$

Where $Q$ is the target PTC and $Q^{\prime}$ is the converted PTC. The measurement results are shown in Figure 6 (a), the two PTC has been converted to the same coordinate system, but still cannot overlap. By using the ICP algorithm, the feature points have been found and the two PTCs have been merged preciously, as shown in Figure 6 (b). The final PTC data of the spiral curved hollow skeleton is shown in Figure 6 (c) and it contains 24 different scanning frames of the LiDAR camera. In order to compare the deformation of the printed structure with the theoretical geometry, the PTC has been converted to an STL 3D model and imported into RoboDK. It is obvious that the printed skeleton structure has a large deformation. The deformation occurs at the area in which the structure starts to incline to the left side with about a 30 degree angle. As shown in Figure 6 (d), the blue shape is the printed skeleton and the yellow curves are the theoretical paths. The layer-by-layer printed CCFRP cannot maintain the original shape due to lacking supporting structures. The maximum distance between the medial axes of the two skeletons is about $15 \mathrm{~mm}$. Hence, the corrected information of the geometry will be transferred to the second robot to adjust the printing path. The printing path of the lattice structure, which should connect to the hollow skeleton, will be modified to ensure the lattice truss can bond to the joint position on the printed skeleton.
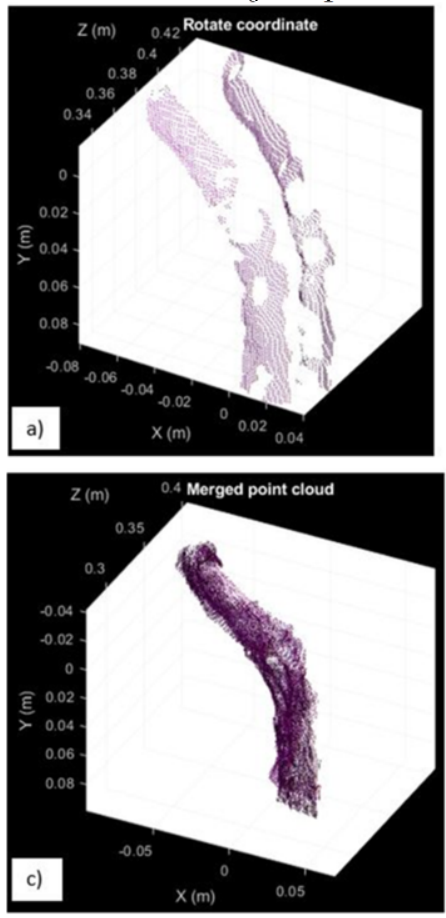
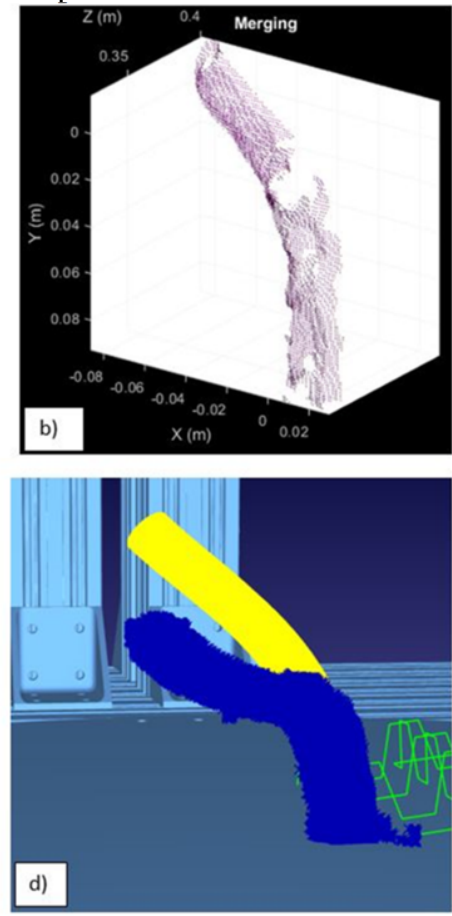

Figure 6. a) PTCs after coordinate transformation, b) PTCs merged by ICP algorithm, c) one side of the 3D reconstructed hollow skeleton that contains 24 frames of PTC data, d) STL model of the skeleton that inputted into RoboDK and compared with theoretical shape 


\section{Conclusions}

In this paper, we present the concept of LiDAR based multi-robot cooperation strategies for the 3D printing of continuous carbon fiber reinforced composite structures. The $3 \mathrm{D}$ printing platform with two UR robots and the corresponding printing heads have been established. By using the LiDAR camera, the geometry of the printed CCFRP structures can be obtained for the cooperation purpose of the second robot. The initial research is conducted in an offline process, which indicates the $3 \mathrm{D}$ scanning works after the $3 \mathrm{D}$ printing process of the UR robot. It shows valuable results to control the robots and adjust the programmed printing paths. With these efforts of robot programming for 3D printing and LiDAR scanning, a closed-loop control of the printing process for continuous carbon fiber reinforced thermoplastics in real-time will be studied and developed in the ongoing project.

\section{References}

[1] Soutis C. Aerospace engineering requirements in building with composites. Polymer Composites in the Aerospace Industry, Woodhead Publishing; 2020: p322. https://doi.org/10.1016/B978-0-08-102679-3.00001-0.

[2] Cheah LW, Cars on a diet: the material and energy impacts of passenger vehicle weight reduction in the U.S., Massachusetts Institute of Technology, 2010.

[3] Li N, Link G, Ting W, Ramopoulos V, Neumaier D, Hofele J, Walter M, Jelonnek J. Path-designed 3D printing for topological optimized carbon fiber composite structures. Composite Part B. 2020; 182:1-5.

[4] Li N, Li Y, Liu S. Rapid prototyping of continuous carbon fiber reinforced polylacticacid composites by 3D printing, Journal of Material Processing Technology. 2016; 238:218-225.

[5] Wang T, Li N, Link G, Jelonnek J, Fleischer J, Dittus J, Kupzik D. Load-dependent path planning method for $3 \mathrm{D}$ printing of continuous fiber reinforced plastics. Composites Part A. 2021;140:1-11.

[6] De Backer W., Bergs AP, Van Tooren MJ. Multi-axis multi-material fused filament fabrication with continuous fiber reinforcement. In 2018 Structural Dynamics, and Materials Conference. 2018; p91.

[7] Kabutan R, Tanaka R, Oomori S, Morita M, Inohira E, Yoshida K, Ohtake H, Nishida T. Development of robotic intelligent space using multiple RGB-D cameras for industrial robots. ICT-ROBOT, Busan, Korea, September, 2016.

[8] Cueva CWF, Torres SHM, Kern MJ. 7 DOF industrial robot controlled by hand gestures using microsoft kinect v2. In Proceedings of the 2017 IEEE 3rd Colombian Conference on Automatic Control, CCAC 2017-Conference Proceedings, Cartagena, Colombia, October 2017; pp 1-6.

[9] Wang L, Liu S, Liu H, Wang XV. Overview of human-robot collaboration in manufacturing. In 5th International Conference on the Industry 4.0 Model for Advanced Manufacturing, AMP 2020; Belgrade; 2020 June,15-58p. Springer. 